\title{
Comparison of the Diagnostic Efficacy of Rapid Immunochromatographic Test with Microwell ELISA in the detection of Dengue NS1 Antigen - A Retrospective Study
}

\author{
S. Krithikaa*, Marquess Raj, Silambarasi, Prabhu and Pramila
}

Department of Microbiology, Apollo Diagnostics, Regional Reference Laboratory (Apollo Health \& Lifestyle Ltd.), No:6, 9th Avenue, Rathan Building, Ashok Nagar, Chennai-600083

*Corresponding author

\begin{tabular}{|c|c|}
\hline & A B S T R A C T \\
\hline Keywords & \multirow{10}{*}{$\begin{array}{l}\text { Dengue fever is a Flavirus disease transmitted by Aedes mosquito. Primary dengue virus } \\
\text { infection is characterized by elevations in specific NS1 antigen levels } 0 \text { to } 9 \text { days after the } \\
\text { onset of symptoms. This retrospective study was conducted to assess performance of rapid } \\
\text { ICTs in acute dengue diagnosis in comparison to ELISA test. It was conducted on } 2234 \\
\text { serum samples received at The Department of Microbiology, Apollo Diagnostics, Regional } \\
\text { Reference Laboratory, Chennai from September } 2017 \text { to November } 2017 \text { by ICT and } \\
\text { ELISA to detect NS1 antigen. Total NS1 positive samples were } 763(34.1 \%) \text { by Rapid ICT } \\
\text { and } 815(36.5 \%) \text { by ELISA. Five hundred and thirteen (513-63\%), } 234(28.7 \%) \text { and } \\
214(26.3 \%) \text { of the positive samples belonged to male, female and children respectively. } \\
\text { Monthly analysis of the data during the study period showed that } 318(39.2 \%), 383(46.9 \%) \\
\text { and } 114(13.9 \%) \text { of the dengue NS1 antigen were detected during the months of September, } \\
\text { October and November } 2017 \text { respectively. The sensitivity of Dengue NS1 Rapid ICT when } \\
\text { compared with ELISA was found to be } 93.6 \% \text { and specificity was found to be } 100 \% \text {. } \\
\text { ELISA has a minimal edge over Rapid ICT in the detection of Dengue NS1 antigen. Hence } \\
\text { ICT in combination with ELISA could help in early and accurate diagnosis of acute } \\
\text { dengue infection. }\end{array}$} \\
\hline & \\
\hline $\begin{array}{l}\text { Immuno } \\
\text { chromatographic }\end{array}$ & \\
\hline Test (ICT), Enzyme & \\
\hline & \\
\hline & \\
\hline & \\
\hline $\begin{array}{l}\text { Sensitivity, } \\
\text { Specificity }\end{array}$ & \\
\hline Article Info & \\
\hline $\begin{array}{l}\text { Accepted: } \\
\text { 20 December } 2017 \\
\text { Available Online: } \\
\text { 10 January } 2018\end{array}$ & \\
\hline
\end{tabular}

\section{Introduction}

Dengue fever which is caused by DENV and belongs to the Flavivirus genus of the Flaviviridae family ${ }^{1}$. It is an arthropod-borne viral infection transmitted to humans by mosquitoes, primarily Aedes aegypti and Aedes albopictus and is endemic to both tropical and sub-tropical regions of the world ${ }^{2}$. The disease has become global public health concern because the infection incidence rates have increased 30 -fold in the past 50 years. WHO has placed India under Category A as Dengue is endemic in our country ${ }^{3}$.

DENV is a positive-sense, single-stranded RNA virus. The genome encodes three structural proteins - the capsid (C), membrane (M) and envelope (E) glycoproteins - as well as seven non-structural proteins (NS1, NS2A, 
NS2B, NS3, NS4A, NS4B and NS5). There are four distinct DENV serotypes (DENV-1 4), all of which are now circulating in Asia, Africa and the Americas ${ }^{4}$.

After an incubation period of $4-8$ days, infection can range from mild dengue fever (DF) to severe forms of DENV infection, referred to as dengue hemorrhagic fever (DHF) and dengue shock syndrome (DSS) ${ }^{5}$. In 2009, the WHO introduced a new classification system for DENV, replacing the traditional DF and DHF/DSS with DENV infection with and without warning signs and severe DENV infection. Asymptomatic dengue cases are mostly seen in children and also in adults with primary infection. Symptoms of dengue are fever, headache, fatigue, body pains, rash, petechiae and bleeding through nose, gums and gastrointestinal tract. Though most patients recover after a self-limiting illness, about 5 $10 \%$ of patients progress to hypovolemic shock with respiratory distress, pleural effusion, pericardial effusion, rising haematocrit values, thrombocytopenia etc. If untreated, the mortality of patients with acute DENV infection can be as high as $20 \%$, although appropriate case management can reduce mortality to less than $1 \%^{6}$.

Accurate diagnosis of dengue is an important component of public health surveillance since clinical diagnosis does not differentiate dengue from other diseases that present with dengue-like signs and symptoms (e.g., malaria, leptospirosis, measles, influenza, Japanese encephalitis, West Nile fever, yellow fever $)^{7}$. Hence, there is the global need for accurate dengue diagnostics. There are three main laboratory methods to diagnose dengue infection: viral isolation in culture, detection of viral RNA, NS1 antigen and specific $\operatorname{IgM} / \operatorname{IgG}$ antibodies in paired sera. The gold standard is usually a combination of these methods ${ }^{8}$. However, methods such as virus isolation and genomic RNA detection (PCR), need a specialized laboratory, well trained laboratory personnel and costly, which are not widely available in resource limited settings. Primary dengue virus infection is characterized by elevations in specific NS1 antigen levels up to $50 \mu \mathrm{g} / \mathrm{ml} 0$ to 9 days after the onset of symptoms; this generally persist upto 15 days ${ }^{9}$. As per Indian national guidelines, a patient is labelled as a "probable" if he satisfies the clinical criteria during dengue outbreak or positive non ELISA based immuno-chromatography tests (ICT) such as NS1 antigen(Ag) ICT "confirmed"e when NS1 $\mathrm{Ag}$ is positive by ELISA ${ }^{10}$.

There were many studies conducted to calculate the sensitivity and specificity of the rapid diagnostic tests that are used to detect NS1 Ag and there was a considerable variation in the percentages reported. Hence this retrospective analysis of the available data was conducted to assess performance of rapid ICTs in acute dengue diagnosis in comparison to Microwell ELISA test in terms of sensitivity and specificity.

\section{Materials and Methods}

This retrospective study was conducted on 2234 serum samples received at The Department of Microbiology in Apollo Diagnostics, Regional Reference Laboratory, Chennai from September 2017 to November 2017. All samples were tested using a commercially available immunochromatographic test (ICT) followed by ELISA for NS1 antigen detection.

\section{Detection of NS1 antigen by Immuno- chromatographic Test (ICT)}

After bringing the kit to room temperature, place the device on a flat surface and add $100 \mu 1$ of the serum sample (2 drops) in the sample window. Read the results in 20 minutes. 


\section{Interpretation of results}

1. NEGATIVE: If only one band (Control band) appears in the result area, Interpret the result as negative. This shows that the specimen does not contain Dengue Ns1 antigen.

2. POSITIVE: If two bands (Control \& Test) appear in the result area the specimen is reactive for Dengue NS1 antigen

3. INVALID: If no band appears after the test is complete, interpret the result as invalid. This shows that the test has been performed incorrectly or there was some procedural error. Please check the procedure and retest using a new device.

\section{Detection of NS1 antigen by Microwell ELISA}

Fit the strip holder with the required number of Anti-Dengue NS1 antibody coated strips. Arrange the assay control wells in a horizontal or vertical configuration. Configuration is dependent upon reader software. Add $50 \mu \mathrm{l}$ Diluent in all the wells. Add $50 \mu 1$ Negative Control in A-1well, $50 \mu$ Calibrator in B-1, C1 and D-1 well, $50 \mu$ Positive Control in E-1 well and $50 \mu \mathrm{l}$ sample in F-1 well onwards. Add $100 \mu \mathrm{l}$ of working Conjugate Solution in each well. Ensure thorough mixing of controls, samples to be tested and working conjugate to get reproducible results. Apply cover seal. Incubate at $37^{\circ} \mathrm{C}+1^{\circ} \mathrm{C}$ for $90 \mathrm{~min}$. $+1 \mathrm{~min}$. While the samples and working Conjugate are incubating, prepare working Wash Solution as specified in preparation of reagents. Take out the plate from the incubator after the incubation time is over and, wash the wells 6 times with working Wash Solution. Add $150 \mu \mathrm{l}$ of working substrate solution in each well. Incubate at room temperature (20$30^{\circ} \mathrm{C}$ ) for $30 \mathrm{~min}$. in dark. Add $100 \mu \mathrm{l}$ of stop solution. Read absorbance at $450 \mathrm{~nm}$. within 30 minutes in ELISA Reader. Calculate the
Dengue NS1 antigen units by multiplying the sample O.D. ratio by 10 .

\section{Interpretation of results}

a. If the Dengue NS1 Ag Units is $<9$ then interpret the sample as Negative for Dengue NS1 Antigen.

b. If the Dengue NS1 Ag Units is between 9 11 then interpret the sample as Equivocal for Dengue NS1 Antigen.

c. If the Dengue NS1 $\mathrm{Ag}$ Units is > 11 then interpret the sample as Positive for Dengue NS1 Antigen.

\section{Results and Discussion}

Of the 2234(100\%) samples tested for NS1 antigen by Rapid Immuno-chromatographic test (ICT), 763(34.1\%) showed positive for the presence of NS1 antigen and 1471(65.9\%) were found to be negative. When the samples tested for NS1 antigen by ELISA, 815(36.5\%) showed positive for the presence of NS1 antigen whereas $1419(63.5 \%)$ were found to be negative. The sensitivity of Dengue NS1 Rapid ICT when compared with ELISA was found to be $93.6 \%$ and specificity was found to be $100 \%$ (Table 1: Study Results and Comparison of Rapid ICT with ELISA).

Demographic analysis of the NS1 antigen positives showed that 513(63\%), 234(28.7\%) and $214(26.3 \%)$ of the positive samples belonged to male, female and children respectively. Our study showed a male preponderance in concordance with the studies conducted by Gupta et $a l^{11}$., 2006; Chakravarti et $a l^{12}$, 2005; Sarkar et $a l^{13}$., 2012 and a greater prevalence among the adult population. Ankita Nisarta et l $^{14}$ reported that females were more commonly affected than males with male to female ratio of $1: 1.35$ in contrast to our study (Figure 1: Demographic analysis of the positive samples). 
Table.1 Study results and comparison of rapid ICT with ELISA

\begin{tabular}{|c|c|c|c|c|}
\hline & $\begin{array}{c}\text { Detection of NS1 } \\
\text { Antigen by Rapid } \\
\text { ICT }\end{array}$ & $\begin{array}{c}\text { Detection of NS1 } \\
\text { Antigen by } \\
\text { ELISA }\end{array}$ & & \\
\hline NS1 Antigen Positive & $763(34.1 \%)$ & $815(36.5 \%)$ & Sensitivity & $93.6 \%$ \\
\hline NS1 Antigen Negative & $1471(65.9 \%)$ & $1419(63.5 \%)$ & Specificity & $100 \%$ \\
\hline Total & $2234(100 \%)$ & $2234(100 \%)$ & & \\
\hline
\end{tabular}

Figure.1 Demographic analysis of the positive samples

\section{Percentage Distribution}

Children,
$26.30 \%$
Female, $28.70 \% \quad$ Male, $63 \%$

Male Female Children

Figure.2 Month wise distribution of dengue NS1 antigen positives during the study period

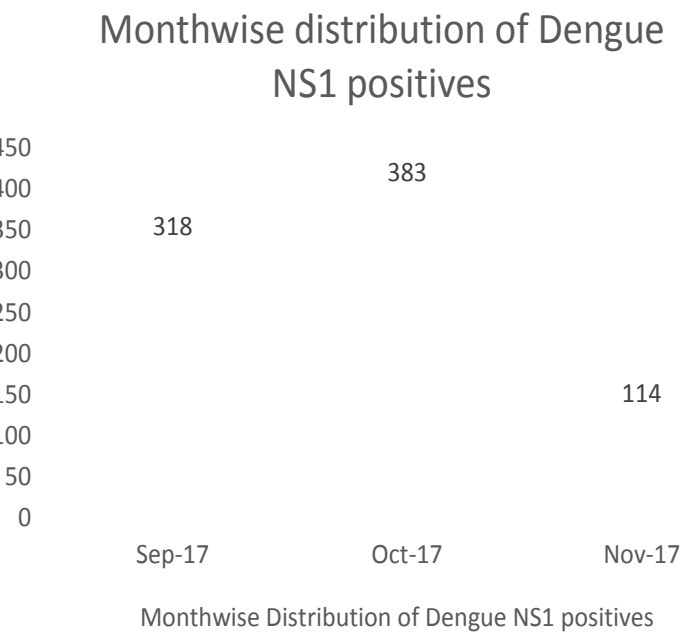

The prevalence of NS1 antigen was found to be $36.5 \%$ from our study which is similar to the studies conducted by J.V. Sathish et $a l^{15}$ $(36.34 \%)$ in 2017 and Priyadharshini
Shanmugam et al ${ }^{16}$ (38.4\%) in 2016. Paradesi Naidu et $a l^{17}$ in 2015 reported a lower prevalence of $12.5 \%$ when compared to our study. 
From the monthly analysis during the study period we observed that 318(39.2\%), $383(46.9 \%)$ and $114(13.9 \%)$ of the dengue NS1 antigen were detected during the months of September, October and November 2017 respectively. This showed that there was an increase in the incidence of dengue during the month of September 2017 and attained a peak during October and significant decline was seen in November.

Similar observation was also made by Ilamani et $\mathrm{al}^{18}$ (2014) and Priyadharshini Shanmugam et $a l^{16}$ (2016). This was related to the onset of monsoon in South India when the vectors are found in large numbers (Figure 2: Monthwise Distribution of Dengue NS1 antigen positives during the study period).

The sensitivity of Rapid ICT when compared to ELISA was found to be $93.6 \%$ and specificity of $100 \%$ which corroborates with the results of Mahesh Reddy et $a l^{19}$ in 2016 (90.1\% sensitivity and $98.45 \%$ specificity). Similar results were also observed by Groen et $a l^{20}$., (2000) and Shih et $a l^{21}$ (2016). Whereas Subhamoy Pal et $a l^{22}$ (2014) reported that sensitivity ranges from $71.9 \%$ $79.1 \%$. This shows that ELISA is more sensitive and specific in detecting NS1 antigen. NS1 antigen detection assay has an advantage of detecting infection very early (from DAY 1), however it disappears early and is of little use in the early convalescence phase when antibody detection becomes useful ${ }^{23}$.

To conclude, ELISA has a minimal edge over Rapid ICT in the detection of Dengue NS1 antigen. Hence NS1-antigen detection through ICT in combination with ELISA could help in early and accurate diagnosis of dengue infection in the laboratories where facilities and trained staff are available. Immunochromatographic test being cost effective, and needs less technical expertise can be used in peripheral health care centres for mass screening activities or in laboratories that have limited resources for the detection of dengue fever. But ELISA is recommended for further confirmation because of its high sensitivity and specificity and specificity.

\section{Acknowledgement}

We the authors gratefully acknowledge the management authorities for permitting us to conduct this study.

\section{References}

1. Guzman M et al., Dengue: a continuing global threat. Nat Rev Microbiol. 2010; 8: S7-16.

2. Dengue and the Aedes aegypti mosquito. Atlanta, GA: CDC; 2012.

3. Global strategy for dengue prevention and control. Geneva: WHO; 2012 - 2020.

4. Zhang $\mathrm{B}$ et al., Diagnosing dengue virus infection: rapid tests and the role of micro/nanotechnologies. Nanomedicine: NBM. 2015; 11: 1745-1761.

5. Guzman MG and Harris E. Dengue. Lancet. 2015; 385:453-65. doi: 10.1016/S01406736(14)60572-9. Epub 2014 Sep 14

6. WHO, Dengue haemorrhagic fever: diagnosis, treatment, prevention and control $2^{\text {nd }}$ edition. Geneva: World Health Organization. [Internet]. WHO [cited 2012 May 6]; Available from: http://www.who.int/csr/resources/publications/d engue/ Dengue publication/en/.

7. Makroo RN, Raina V, Kumar P, Kanth RK. Role of platelet transfusion in the management of dengue patients in a tertiary care hospital. Asian J Transfus Sci. 2007;1(1):4-7.

8. Kittigul L, Pitakarnjanakul P, Sujirarat D, Siripanichgon K. The differences of clinical manifestations and laboratory findings in children and adults with dengue virus infection. Journal of Clinical Virology. 2007 Jun 30; 39(2): 76-81.

9. Libraty DH, Young PR, Pickering D, Endy TP, Kalayanarooj S, Green S, Vaughn DW, Nisalak A, Ennis FA, Rothman AL. High circulating levels of the dengue virus nonstructural protein NS1 early in dengue illness correlate with the development of dengue hemorrhagic fever. 
Journal of Infectious Diseases. 2002 Oct 15; 186(8): 1165-8.

10. National Guidelines for Clinical Management of Dengue Fever, Dec 2014.

11. Gupta, Ekta, Lalit Dar, Geetanjali Kapoor, and Shobha Broor. 2006. "The Changing Epidemiology of Dengue in Delhi, India." Virol. J., 3(1): 1-5. http://dx.doi.org/10.1186/1743$422 \mathrm{X}-3-92$

12. Chakravarti, A., R. Kumaria. 2005. "EcoEpidemiological Analysis of Dengue Infection during an Outbreak of Dengue Fever, India." Virol. J., 2. http://dx.doi.org/10.1186/ 1743422X-2-32.

13. Sarkar, Arindam, Debjani Taraphdar, and Shyamalendu Chatterjee. 2012. "Molecular Typing of Dengue Virus Circulating in Kolkata, India in 2010.” J. Trop. Med., 960329.

14. Nisarta A, Ahir H. Study of Seroprevalence of Dengue Virus Infection in a Tertiary Care Hospital in Patan, Gujarat, India. Int. J. Curr.Microbiol. App. Sci. 2016; 5(10):819-24.

15. Sathish, J.V., Mita D. Wadekar and Pooja, C. 2017. Dengue NS1 Antigen for Early Detection of Dengue Infection. Int.J.Curr.Microbiol. App.Sci. 6(10): 2054-2058. doi: https://doi.org/10.20546/ijcmas.2017.610.244

16. Shanmugan $P$, Soundararajan $N$, Ravi V, Venkatesan P. A study on the prevalence of Dengue fever in Kelambakkam in comparison to an earlier study. Indian J Microbiol Res 2016; 3(2):102-106.

17. Paradesi Naidu A et al., Detection of Dengue NS1 by a Comparative Analysis of Panbio Elisa and Rapid Diagnostic Test Sch. J. App. Med. Sci., Mar 2017; 5(3B): 816-820.

18. Illamani $\mathrm{V}$ et al., Serological Analysis of Clinically Diagnosed Dengue Virus Infection by Immunochromatography., March - April 2014 RJPBCS 5(2): 703-706

19. Mahesh Reddy, R., Kavita Sahai, Ajay Mallik, S. Shoba and Anurag Khera. 2016. Comparative Analysis of Rapid Dengue Testing and ELISA for NS1 Antigen and IgM in Acute Dengue Infection. Int.J.Curr.Microbiol.App.Sci. 5(10): 931-937. doi: http://dx.doi.org/10.20546/ijcmas. 2016.510.100

20. Groen, Jan, et al., 2000. "Evaluation of Six Immunoassays for Detection of Dengue VirusSpecific Immunoglobulin M and G Antibodies." Clin. Diag. Lab. Immunol., 7(6): 867-71.

21. Shih, Hsin-I et al., 2016. "Applications of a Rapid and Sensitive Dengue DUO Rapid Immunochromatographic Test Kit as a Diagnostic Strategy during a Dengue Type 2 Epidemic in an Urban City." PloS one, 11(7): $\mathrm{e} 0158437$.

22. Pal, Subhamoy, et al., 2014. "Evaluation of Dengue NS1 Antigen Rapid Tests and ELISA Kits Using Clinical Samples." PloS one, 9(11): e113411.

23. Pramod S. Manthalkar, B.V. Peerapur. 2017. Utility of NS1 Antigen for Diagnosis of Dengue Virus Infection. Journal of Krishna Institute of Medical Sciences University, 6(1): 72-75.

\section{How to cite this article:}

Krithikaa, S., Marquess Raj, Silambarasi, Prabhu and Pramila. 2018. Comparison of the Diagnostic Efficacy of Rapid Immunochromatographic Test with Microwell ELISA in the Detection of Dengue NS1 Antigen- A Retrospective Study. Int.J.Curr.Microbiol.App.Sci. 7(01): 2654-2659. doi: https://doi.org/10.20546/ijcmas.2018.701.317 